\title{
Smart safety management with ARIADNE SMS: Save Money. Prevent.
}

\author{
W. D. Käppler \\ Fraunhofer FKIE Research Institute for Communication, \\ Information Processing and Ergonomics, Wachtberg, Germany
}

\begin{abstract}
This paper presents the practical result of many years of research into human error and failure in the form of accident and incident risk analyses and prevention: ARIADNE, the integrated safety management system. This is based on the three-level model of accident causation, seeing human errors as accident causes and the product of a network of influences which already embody the risks of error-relevant conditions as accident causes. In ARIADNE, the accident event is structurally classified using 2,000 keys. Risk management assigns probabilities and accident as well as measure costs to errors and causes of error, and suggests preventative measures on a cost-risk basis. Risk changes are predicted with the aim of improving safety, saving resources and cutting costs. Benefits are described and the selected results of 10,000 accident analyses are presented.

Keywords: action, accident analysis, cognition, cost model, descriptor, endangerment error causes, data mining, human error, injury to persons, investigation cost, judgement, keytable, material damage, measures, perception, prevention, probability, quality assurance, risk management, safety management, safety culture, scaling, semantic net, taxonomy, three-level-model of accidents, what-if-risk simulation.
\end{abstract}

\section{Introduction and problem statement}

Safety, for example at work or in traffic, is a central issue for society. Nonetheless, even work is a cause of accidents and damage. On economic grounds and for reasons of liability, as well as from a humanistic point of view, this is no longer acceptable; after all, according to the Brockhaus encyclopedia 
[1] an accident is an unintentional, suddenly occurring event due to external influences such as a knock, fall, etc., causing material damage or damage to life or limb. Accidents, according to Brockhaus, mainly occur in traffic and at work, as well as during leisure activities and in the home.

Brockhaus does not explain why accidents occur in the first place, or why risks exist. A glance at the statistics shows that errant behavior is the cause of 90 percent of all accidents. In final reports, it is then said that the cause of the accident was human error. Public opinion is satisfied: "So nothing could be done about it ..."

Experts are aware that, in the case of highly developed technology, this cannot be the last word, but is merely an admission of abject failure by those in charge. For example, in road traffic, the central focus is on clarifying issues of liability: Whose fault is the accident? Who has to pay for the damage? To answer these questions, errant behavior, e.g. in the form of alcohol consumption or speeding, is tracked down and punished. However, the processes and factors which lead to errant behavior or speeding in the first place remain almost unnoticed.

Safety can hardly be effectively improved in this manner, which is where the concept of human error comes in. Reason [2] and Senders and Moray [3] ask why even experienced operators overlook important information, make bad decisions and react inappropriately. They concentrate their search on errors, and, moreover, see them as the product of a wide-reaching network of influences, made up of different risk conditions.

Thus, this concept applies not only to failure from a legal point of view, but also to concrete actions or decisions in a current situation and, moreover, to discovering related causes of error and boundary conditions. According to this, errors are a natural byproduct of human behavior and occur in a concrete, situational context. This dependence on context is the basis for modern safety management.

This is illustrated by a simple example in Figure 1. Fast driving, in the upper picture, leads to the destination quickly and without an accident, if the boundary conditions are taken into account. In the worst case, greater risks occur due to excessive speeds. However, tracing this back as the cause of the accident in Figure 1, below, hides the causes of the accident.

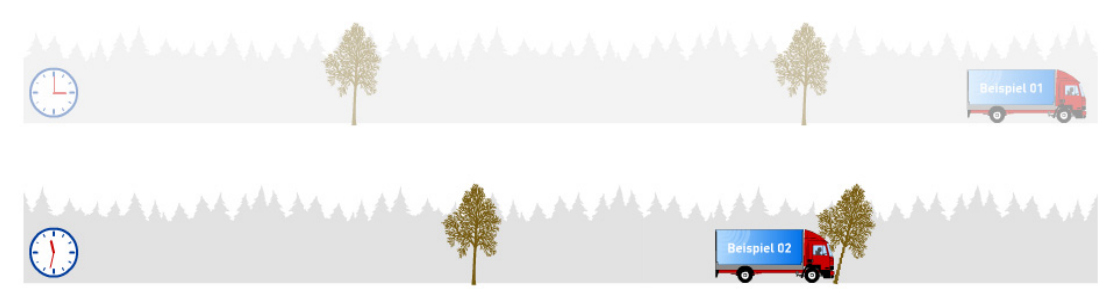

Figure 1: Accident (Käppler et al. [4]). 
In fact, when considered more closely, the error of Running off the Road leads to the accident; excessive speeds and the trees along the avenue worsen the consequences. The question now is therefore: Why was this error made? This simple example only allows speculative answers: Perhaps fatigue or inattentiveness led to errors of perception, and lack of time to speeding.

In the example, fatigue, inattentiveness or speed need to be checked and monitored. However, modern safety management goes far beyond this: It involves the development, implementation and checking of specific measures for prevention. In the example, these are crash barriers, sleep warning devices, or even the removal of the trees or the reduction of time pressure.

\section{Idea and technological implementation}

With this in mind, the three-level model of accident causation in Figure 2 was developed by Käppler [5,6]. According to Frieling and Sonntag [7], in the usual environment of technological, organizational and personal conditions of work organization, for example, there are ample combinations of accident causes. In certain conditions these result in concrete errors, which, in turn, can cause accidents.

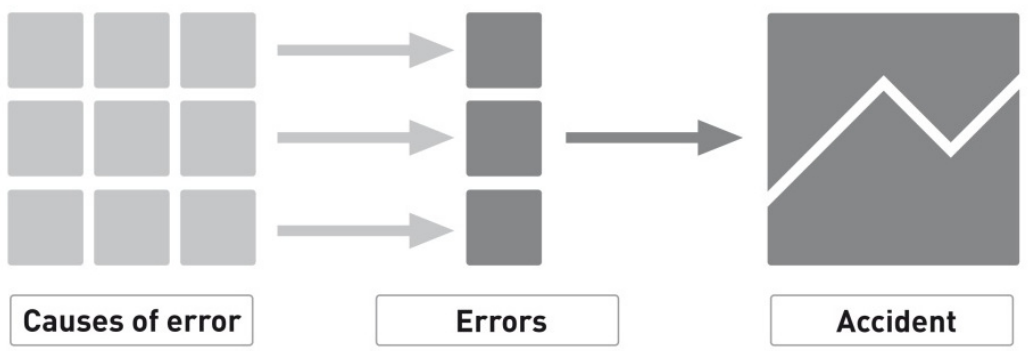

Figure 2: Three-level model of accident causation (Käppler [6]).

With this idea in mind, an empirical investigation was carried out into more than 2,000 accidents during different working activities, including aviation and road traffic. A taxonomy of errors and causes was developed based on Chappelow [8]. Originally, it was divided into errors and technological defects. This duality was discarded, however, as careful analysis showed that defects could also be traced back to errors, e.g. during procurement, construction or manufacturing. That taxonomy describes roughly 150 causes of error in the eight main categories in Figure 3.

- Work organization causes of error are faults in the targeted ordering, regulation and incorporation of tasks and activities into social structures, e.g. in the case of rules, regulations or working materials

- Communication causes of error are faults in verbal and non-verbal processes of information transfer 
- Personnel and qualification causes of error are faults in staff selection, allocation and qualification with regard to the knowledge and skills enabling them to carry out the activity

- Quality management causes of error are faults in regularly testing the quality and nature of results, e.g. in the case of standards, instructions and manuals

- Attitude causes of error are constraints on workers' ability to judge subjects and objects consistently in a certain manner, caused by preferences or predispositions, e.g. due to a need for recognition

- Physiology causes of error are constraints on workers caused by life processes such as growth or illness

- Behavior causes of error are observable flaws in workers' actions and decisions. This includes processes of experience which workers are aware of to a greater or lesser degree

- Environmental conditions causes of error are characteristics of the environment which can be described using physical data and which increase risks.

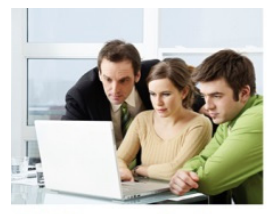

Working organization

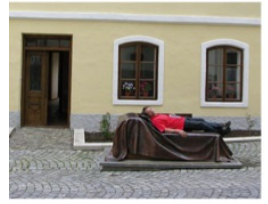

Attitude

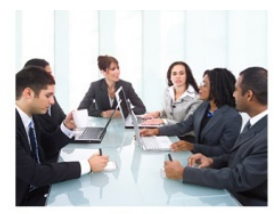

Communication

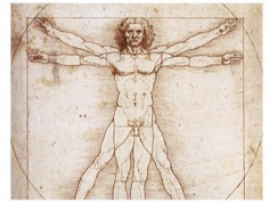

Physiology

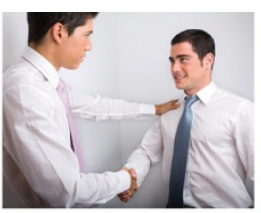

Personnel \& qualification

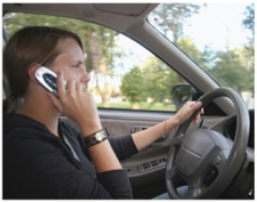

Behavior

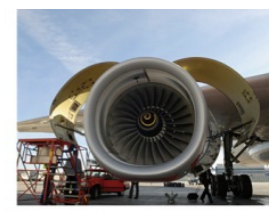

Quality Management

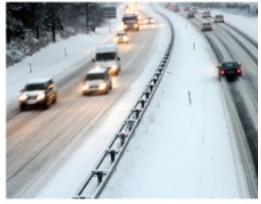

Environment

Figure 3: $\quad$ Categories of error causes (Käppler [6]).

Figure 4 shows the classification of the 35 types of error observed into three categories:

- $\quad$ Errors of perception are a lack of ability to derive from sensory information a comprehensive, sufficient representation of characteristics of the physical and social environment

- Errors of cognition are when plans are formulated based on the correct perception of relevant information, but do not stand up to the requirements of the task and situation

- Errors of action are when a correct plan of action is carried out wrongly; i.e. when sequences of actions are mixed up or the worker falls into similar, habitual routines. 


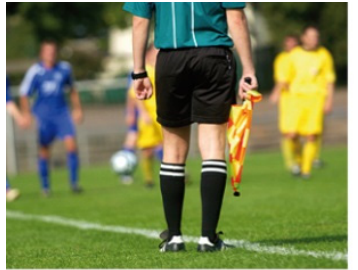

Errors of perception

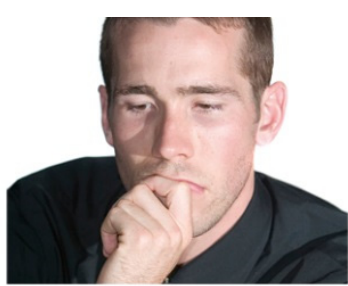

Errors of cognition

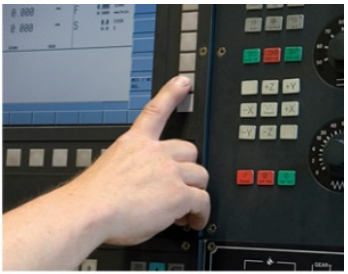

Errors of action

Figure 4: $\quad$ Categories of error (Käppler [6]).

Error identification is often based on indications and it is sometimes unclear whether an error actually set off the accident; for example, a failure to act may merely increase risks or consequent damage. Thus, the likelihood of errors occurring and the effect they have differs. This likelihood can be estimated following the error identification. For scaling, an interval scale was developed with five equal intervals, following Rohrmann [9] and Käppler [10] (see Figure 5).

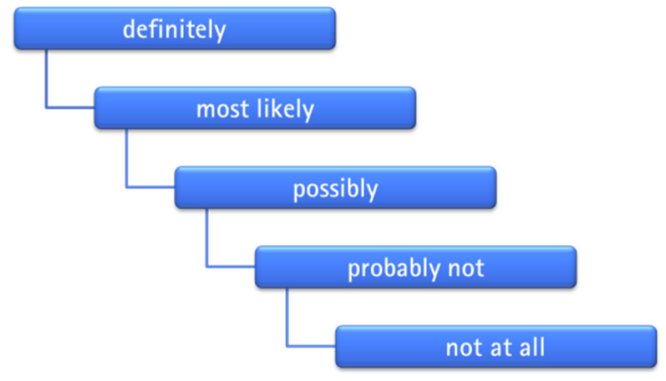

Figure 5: Scaling of errors regarding occurrence and initiation.

If, for example, errors which trigger accidents are more highly rated than those which "only" aggravate the consequences, the scale produces "chains of errors". The identified causes of the accident are also rated in the same way as the errors and developed into chains.

In safety management, knowledge of the errors and their causes, with the scale and chains of effect, allows risk management to be carried out. Risk management is understood as a form of safety culture which sees risks as a lack of information on target achievement, and which aims to reduce these risks. It takes place in these phases:

- $\quad$ Risk identification

- Risk analysis and simulation

- Risk policy.

In Phase 1, using complex mathematical models, the risks of error and causes of error are identified using probabilities and loss expenses. 
In Phase 2, the risk simulation follows, to look into the cause and effect relationship in the case in hand. Using "what-if" simulation, the occurrence and effect of individual causes and errors are manipulated and hypotheses formulated on whether accidents and damage will fail to occur after possible preventive measures are carried out. Simulations of different prevention scenarios show changes in the risks and expenses, and predict potential savings and safety benefits (see Figure 6).

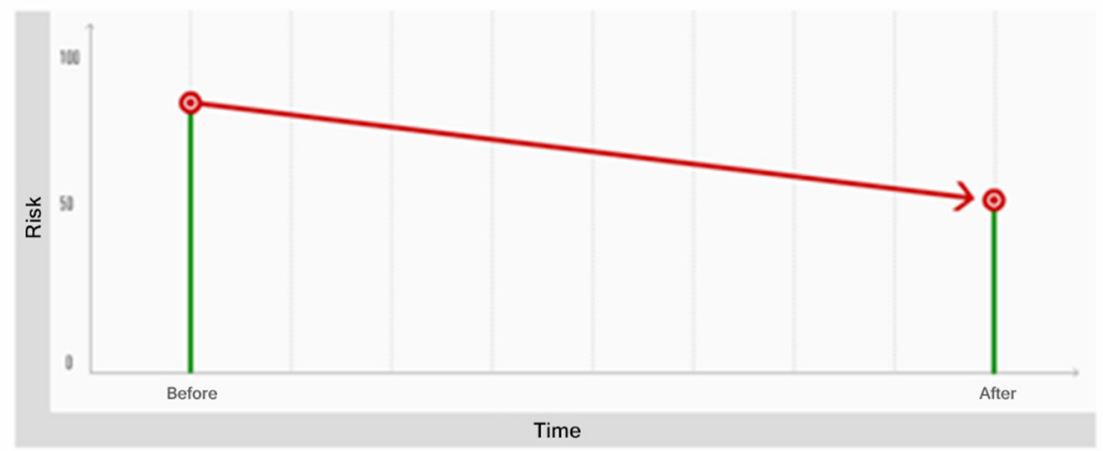

Figure 6: Risk before and after the introduction of preventive measures (Käppler et al. [4, 11]).

Finally, in Phase 3, decisions about risk policy measures are made and implemented to avoid, reduce and distribute risks, build up a reserve to cover risks, prevent losses or pass on costs. One part of the risk policy is quality assurance, a running check on the measure's costs and benefits. This overall concept is today summed up with the term safety management. It is a practical expression of the predominating safety culture according to INSAG [12] and Hudson [13] distinguishes between five levels of increasingly mature safety culture (see Figure 7).

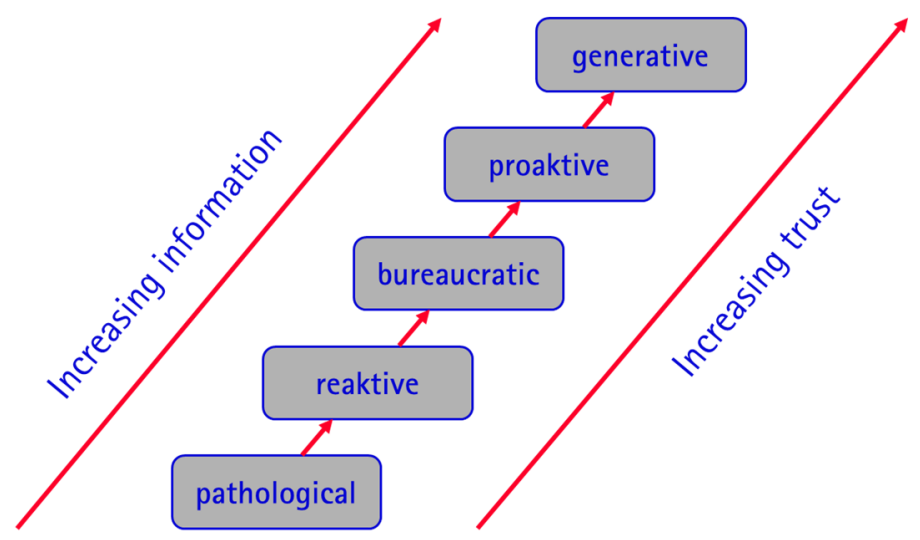

Figure 7: Safety Culture Maturity Model (Käppler et al. [4, 11]). 
- Pathological: Based on the idea, today seen as pathological, that reasonable people do not make any mistakes, along the lines of Palmstrom: “... since, he argues reasonably, that cannot be what must not be" (Morgenstern [14])

- Reactive: Recognizes that safety is a problem and acts after incidents occur

- Bureaucratic: Characterized by hierarchical communication and the bureaucratic administration of large quantities of data. Typical conclusion: "So nothing could be done about it."

- Proactive: Characterized by democratic communication and involves the workers' level. Preventive measures are implemented due to risk or hazard analyses, without damage having occurred

- Generative: Sees safety as an indispensable part of business, e.g. alongside the maximization of profits, and depends upon the active participation of all staff at all levels of organization: strategy, management and work (see Figure 8), (INSAG [12]).

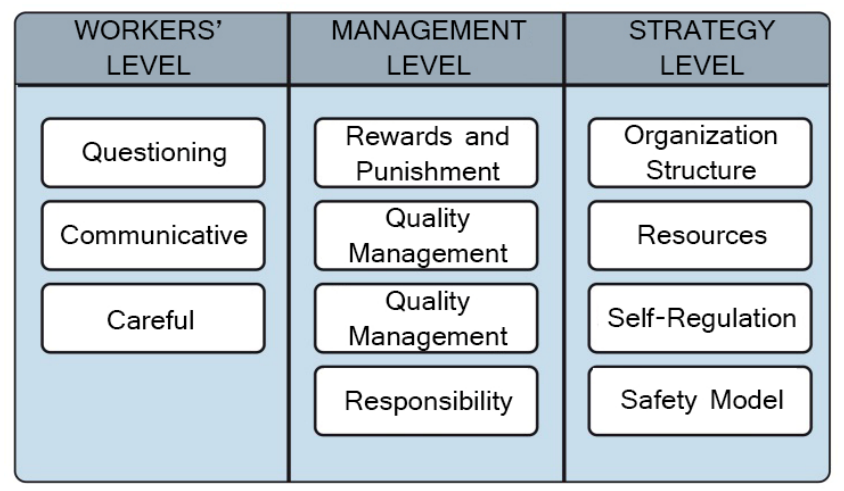

Figure 8: Safety culture and organization (Käppler et al. [4, 11]).

Modern safety management is proactive and promotes a generative safety culture. At the level of the workers, careful questions are asked; at the managerial level, decisions are made in democratic processes; as well as quality management and monitoring, incentives are used to encourage people to report their own errors. On the level of strategy, models are created as targets, for example as regards saving resources.

Safety management sees preventive measures as barriers formed by technology, people or organization to prevent accidents or reduce their results, despite errors occurring. Reason [2] modeled the remaining risks as holes in a Swiss cheese; Figure 9 shows a preventive situation which is "full of holes".

Safety management combines individual measures such that at least one barrier shown in the figure prevents the accident; for example, training measures can reduce the consequences of accidents caused by operating errors. 


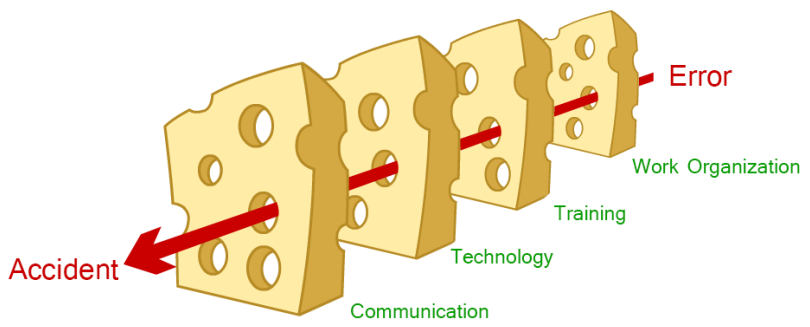

Figure 9: Preventive measures as barriers (Käppler et al. [4, 11]).

\subsection{ARIADNE safety management system}

The ideas outlined above have been joined together to form an integrated safety management system (SMS) for hazards and accidents at work, and put into practice as the ARIADNE web application (see Figure 10). Worldwide access via an internet browser permits:

- Multiple, parallel use by any number of people

- Detailed documentation of how the accident occurred, its consequences and costs

- Analysis of errors and causes

- Risk management and quality assurance.

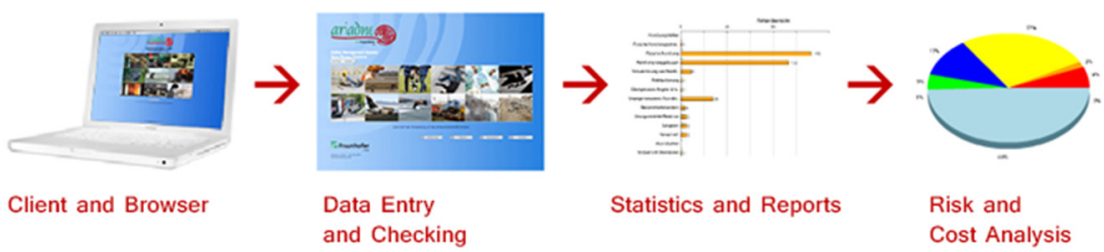

Figure 10: The ARIADNE web application (Käppler et al. [4, 11]).

\subsection{Detailed documentation}

First, accident and hazard data are collected in the database or offline, using a notebook at the scene of the accident; later, they are aligned with the database. Along with the multi-user capability, this ensures flexibility and currency. Up to 2,000 individual items of data are thus documented in the online database (see the example in Figure 11). The individual values are mainly captured by the selection of key values, so as to create a structured database with comparable data. This is made possible by wide-reaching reporting and statistics tools which are available online. 


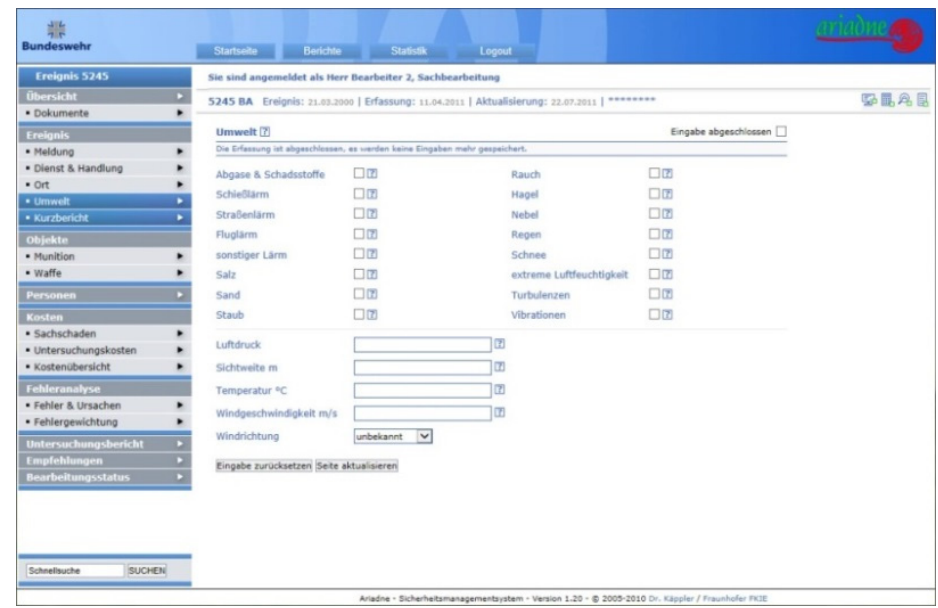

Figure 11: Webpage for capturing the environmental data application in German (Käppler et al. [4, 11]).

\subsection{Accident consequences and costs}

The documentation also includes the consequences and costs of an accident. These are captured in 30 cost accounts as injury to persons, material damage and costs of the investigation (see Figure 12).

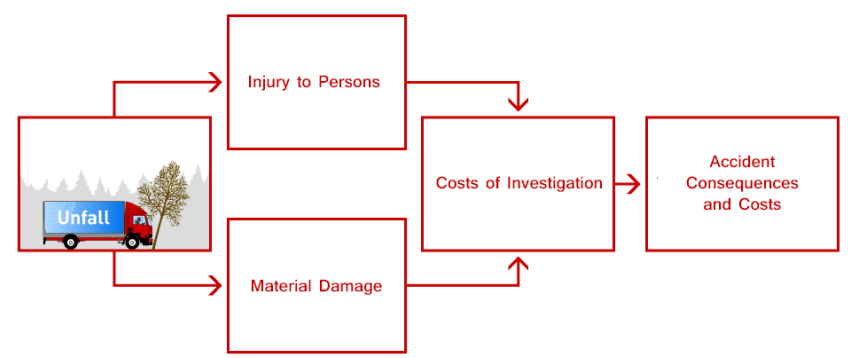

Figure 12: Cost-recording structure application (Käppler et al. [4, 11]).

\subsection{Analysis of errors and causes}

Next, the analysis of errors and causes in Figure 13 is carried out. First, all errors are identified which have caused damage or hazards.

They are weighted and attributed to people. In the same way, causes of error are then identified, weighted and attributed to errors. 


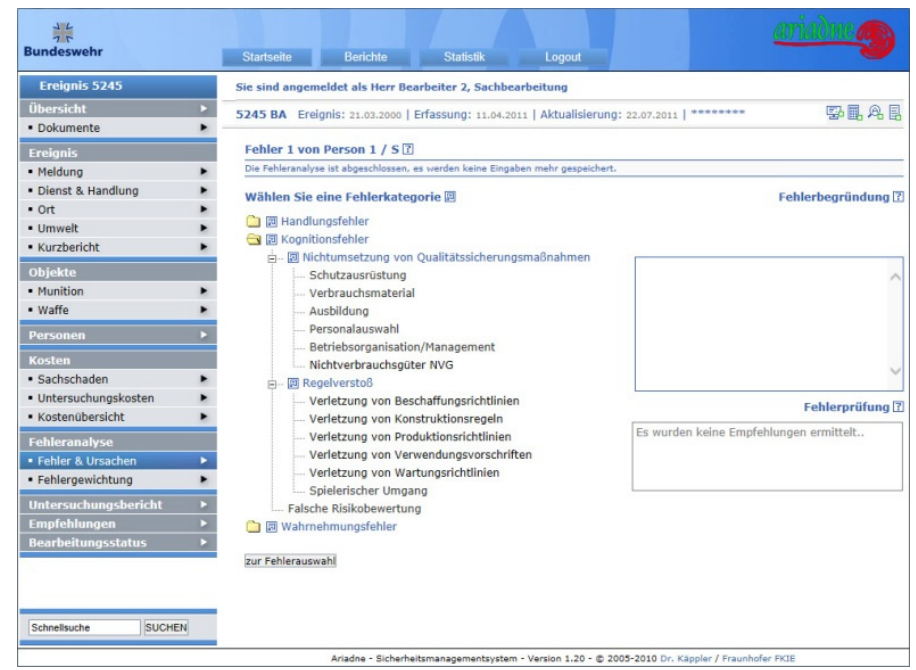

Figure 13: Webpage for error identification in German (Käppler et al. [4, 11]).

\subsection{Risk management}

This extensive management system results in risks, costs and measures. Risk identification shows how likely causes of error are to result in errors, and errors to result in accidents, and proportionally allocates costs to the errors and the causes of error which gave rise to them (see Figure 14).

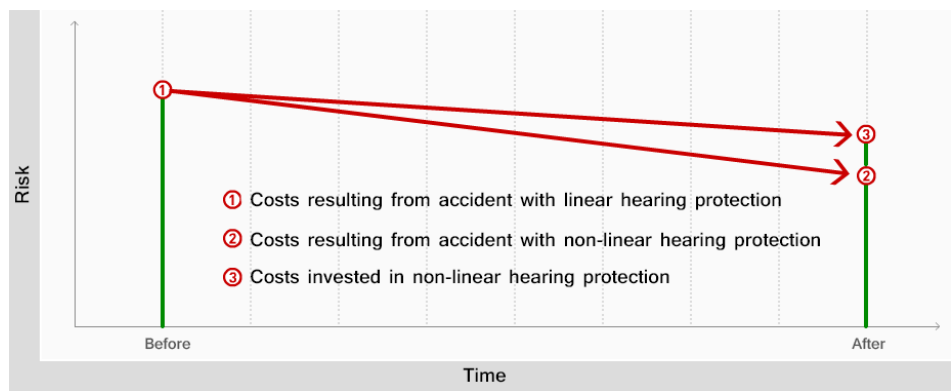

Figure 14: Costs of an accident and preventive measure (Käppler et al. [4, 11]).

"What-If" risk simulation predicts changes in these risks and costs after corresponding preventive measures are introduced. Preventive measures also cost money. These costs are determined and cut back the expected savings, see Figure 14. Detailed descriptions of the mathematical models and processes can be found in Käppler et al. [4], Käppler [5] and Käppler and Dalinger [15]. 


\subsection{Quality assurance}

Preventive measures are documented. The processes and schedules are checked in the SMS quality management, see example in Figure 15. After the start-up period and when a measure has been implemented for the set time, the SMS suggests quality checks and repeating the "before and after" risk analysis, to validate the preventive measure.

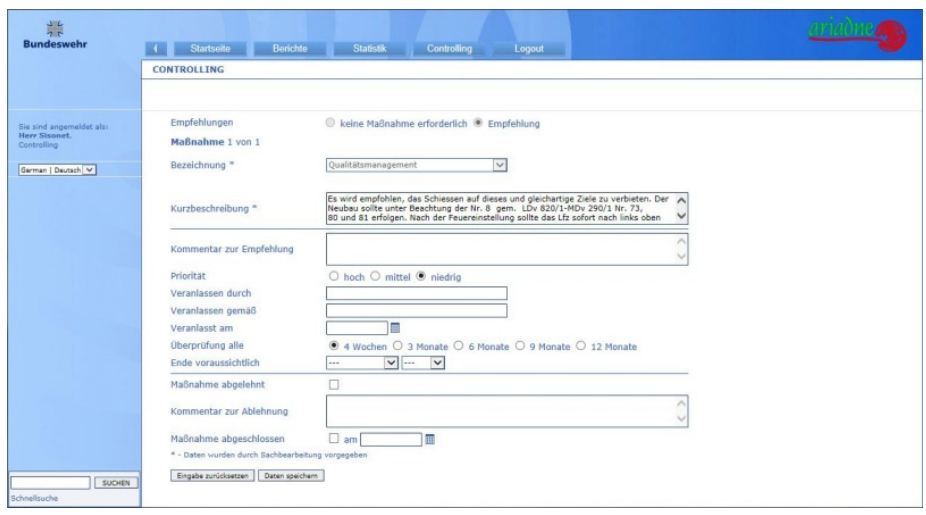

Figure 15: Webpage for quality assurance in German (Käppler et al. [4, 11]).

\subsection{SMS access and security}

The database contains personal data and other information. These are protected against manipulation, unauthorized access and theft using a wide-reaching security concept. Furthermore the SMS manages different access rights for the following groups of users:

- Administration governs the rules and rights of usage, including the key tables, with access to the whole system.

- Analysis has access to all the data material, checks it and carries out evaluations and risk analyses. It runs the development and quality assurance of the application.

- $\quad$ Processing enters data, analyzes the errors and causes of error and suggests preventive measures

- Quality management implements and checks preventive measures

- Guest has read-only access to statistics, analyses and reports

- Audition supervises access and security of the site and data.

\section{Results}

ARIADNE provides information on results online using descriptive statistics, in the form of graphs and tables, mainly using pie charts and histograms. The 
following figures show examples of the results of accident and hazard analyses in the past when handling weapons and munitions in the German armed forces. In these examples it must be noted that data from all incident classes were analyzed together to demonstrate ARIADNE's potential. This is only the first step in developing accurate preventive measures and the basis for identifying trends and weak spots in accident incidents as a whole. Next, the data from the identified problem areas are filtered in detail and evaluated to develop targeted measures. This is shown in detail in Käppler [16] and Käppler et al. [4] also addressing road accidents with motor bikes.

All the following images are downloads from the web application and a data sample recorded in recent years. Altogether, 3,010 incidents were caused by 6,222 errors and 12,419 causes of error. On average, that made two errors and four causes per case in this sample (see Figure 16).

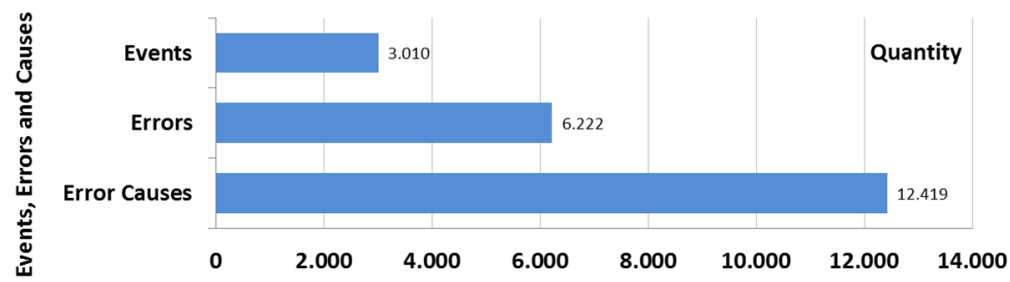

Figure 16: Accidents, errors and causes (Käppler et al. [4]).

\subsection{Documentation}

Figure 17 shows that in the reporting timeframe, more than $€ 26$ million cost in total was caused; on average just under $€ 9,000$ per incident. The lion's share of the costs resulting from the accident goes to injury to persons; only 20 percent are material damage. Investigation costs seem negligible, as according to the information only external costs are calculated, and not, for example, the personnel costs for those processing the case.

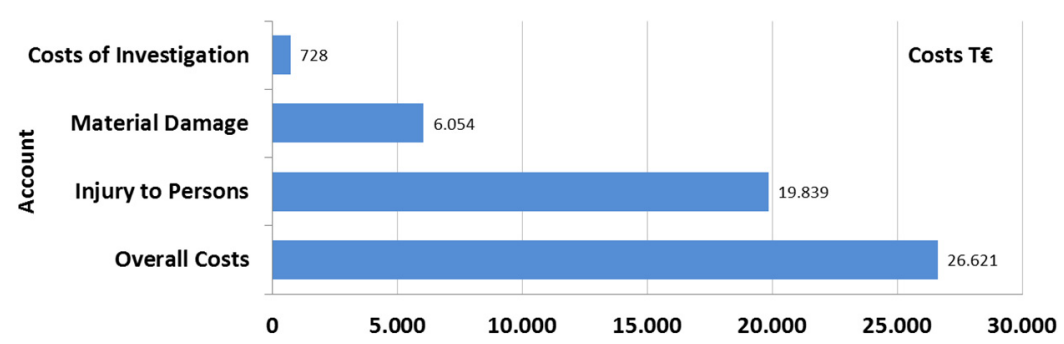

Figure 17: Overview of the costs resulting from accidents (Käppler et al. [4).

Figure 18 shows that just below 10 percent of accidents produced almost 90 percent of all costs resulting from accidents. 
Altogether, 5,926 people were involved, of whom only 146 were female (see Figure 19), that means just 2 percent.

According to Figure 20, 12 soldiers were killed and 50 percent of those involved were injured; 36 percent slightly and 3 percent seriously. In 11 percent

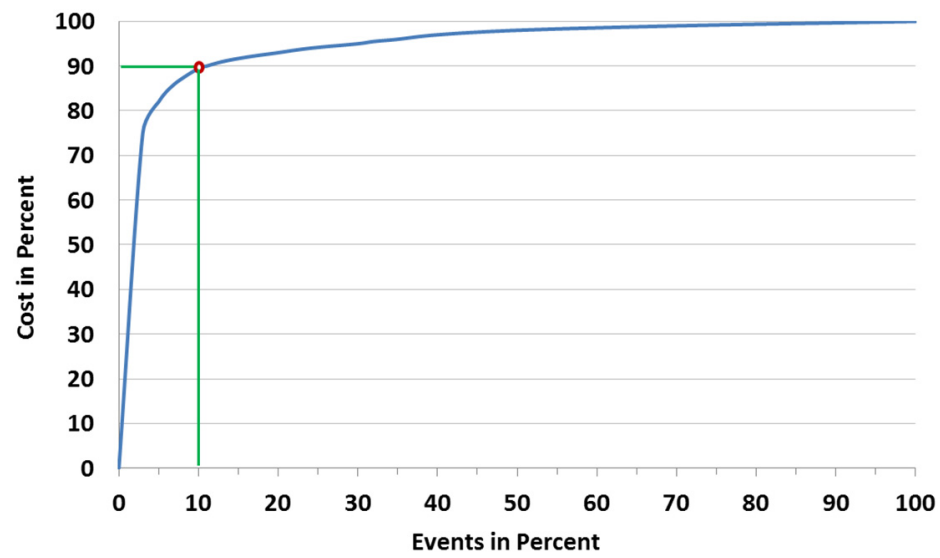

Figure 18: Costs resulting from accidents by number of accidents, in percent (Käppler et al. [4]).

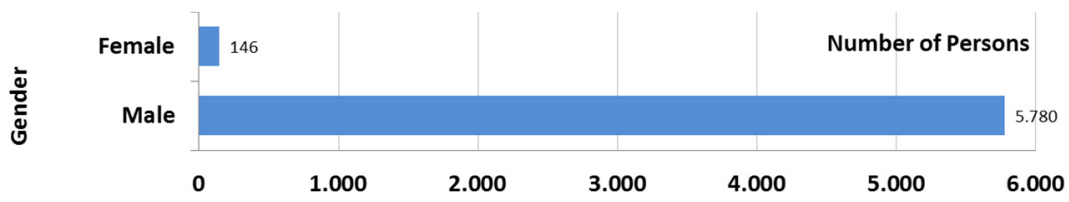

Figure 19: People involved in accidents, by gender (Käppler et al. [4]).

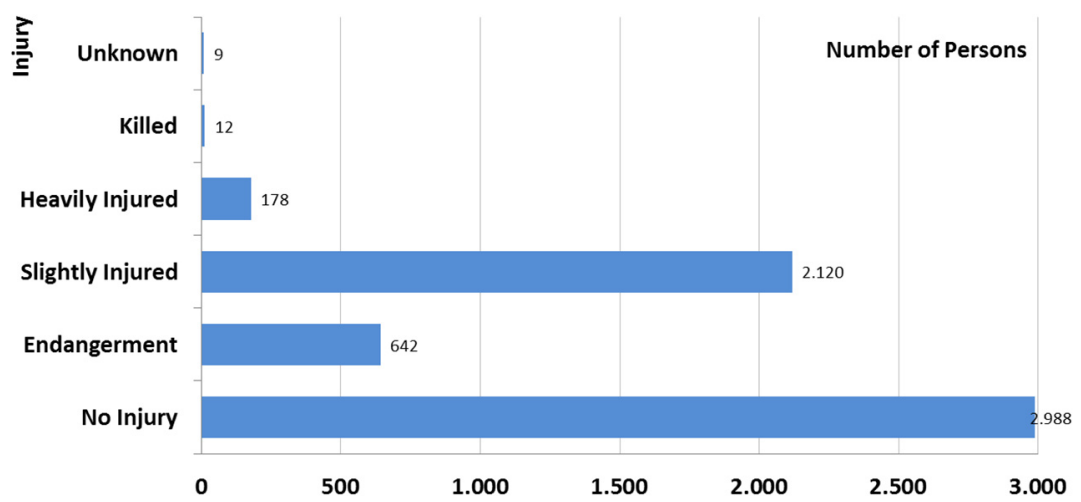

Figure 20: Degree of injury and mortalities (Käppler et al. [4]). 
of cases, people were merely at risk. The proportion of injuries of unknown seriousness relates to technical weapons and munitions complaints where no injuries to people were documented.

Figure 21 shows that accidents were more likely to occur on Tuesdays, Wednesdays and Thursdays, with a peak on Wednesdays. This is typical for accidents at work.

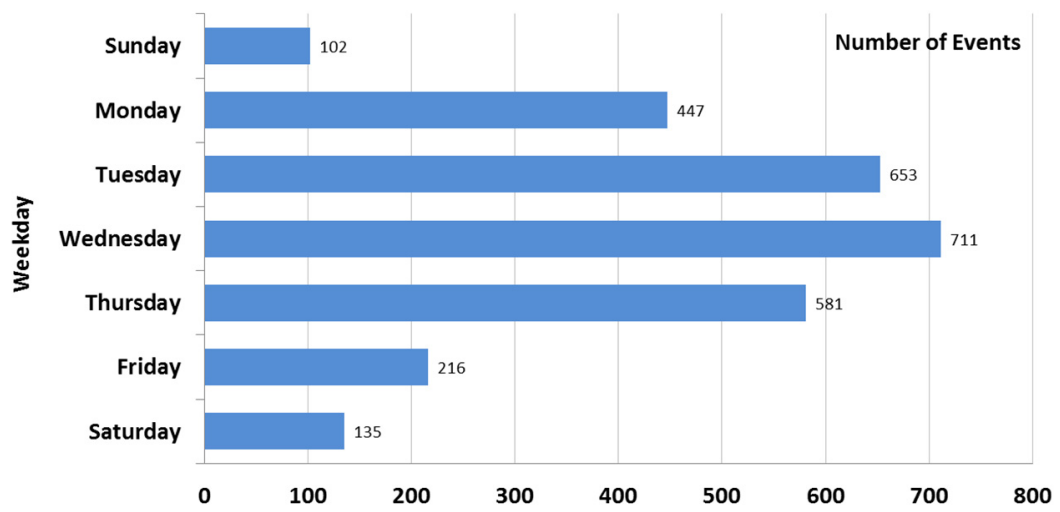

Figure 21: Distribution of accidents across days of the week (Käppler et al. [4]).

\subsection{Analysis of errors and causes}

Sixty percent of the errors causing the event were errors of cognition and 31 percent errors of action. A mere 9 percent of the incidents were traced back to errors of perception (see Figure 22).

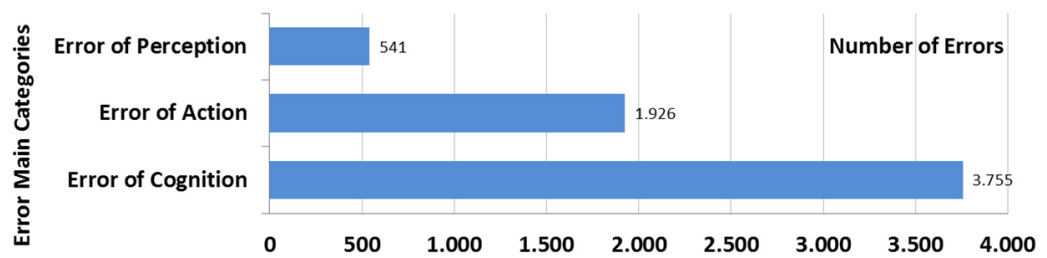

Figure 22: Overview of error main categories (Käppler et al. [4]).

Figure 23 shows details: the most common errors were incorrect operation and actions, followed by the violation of usage regulations, as well as errors in organization and management, incorrect risk evaluation, erroneous use of materials and failure to implement quality assurance measures.

The overview of causes of error in Figure 24 provides further indication of trends and weak spots. The areas of behavior (42 percent), quality management 


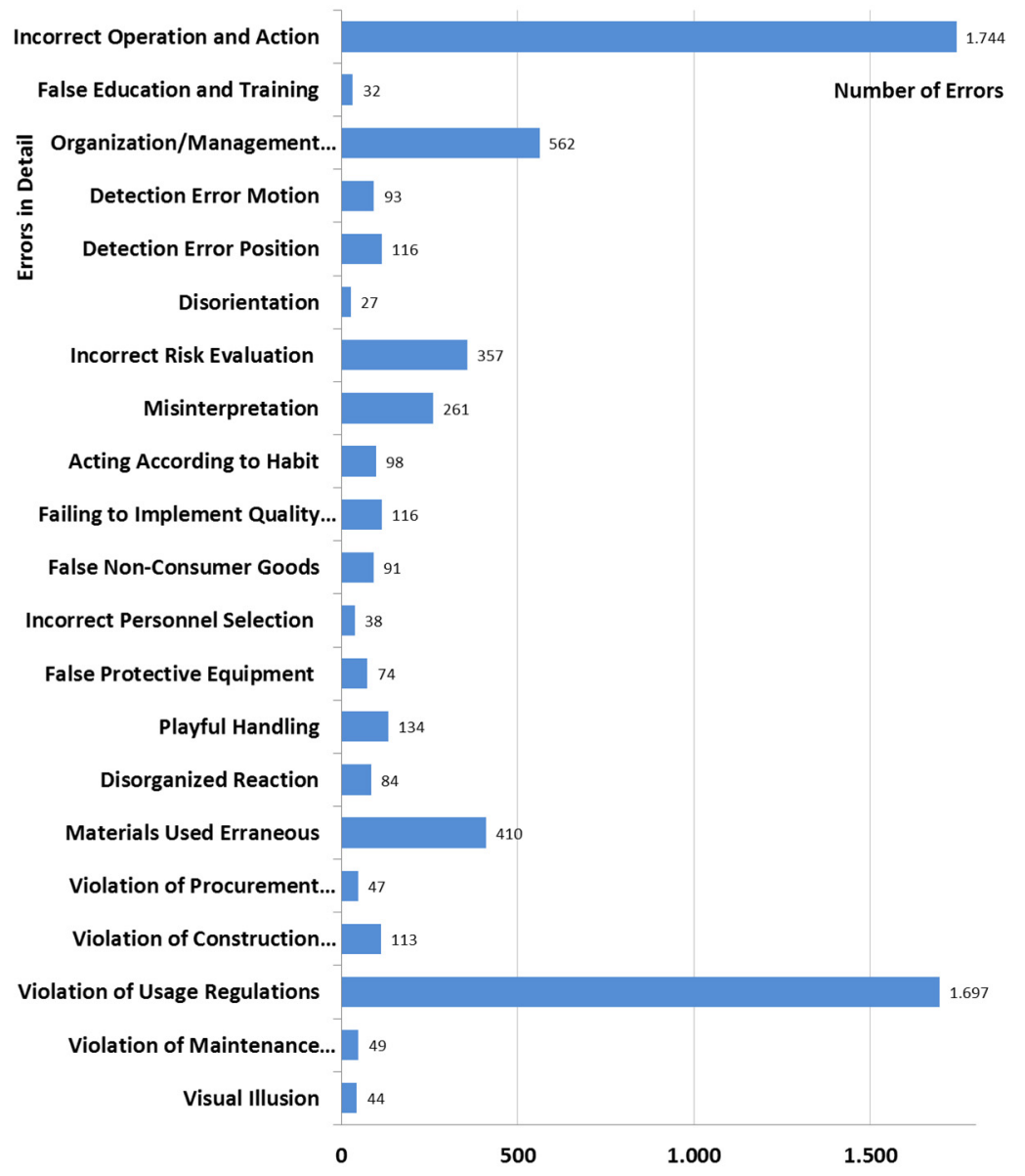

Figure 23: Errors in detail (Käppler et al. [4]).

(22 percent) and personnel and qualification (13 percent) were named the most often. Causes of error in the areas of work organization and attitude were only named in 7 and 10 percent of cases, respectively. Named in between 1 and 2 percent of cases, problems with environmental conditions, communication or physiology played only a minor role as causes of error.

\subsection{Risk management}

However, frequencies do not reveal anything about risks and costs, which are typically discussed in great detail in bureaucratic safety strategies. Formulating preventive measures based on statistical frequencies may be rash and result in ineffective measures, as the past has shown. One aim of a proactive safety 


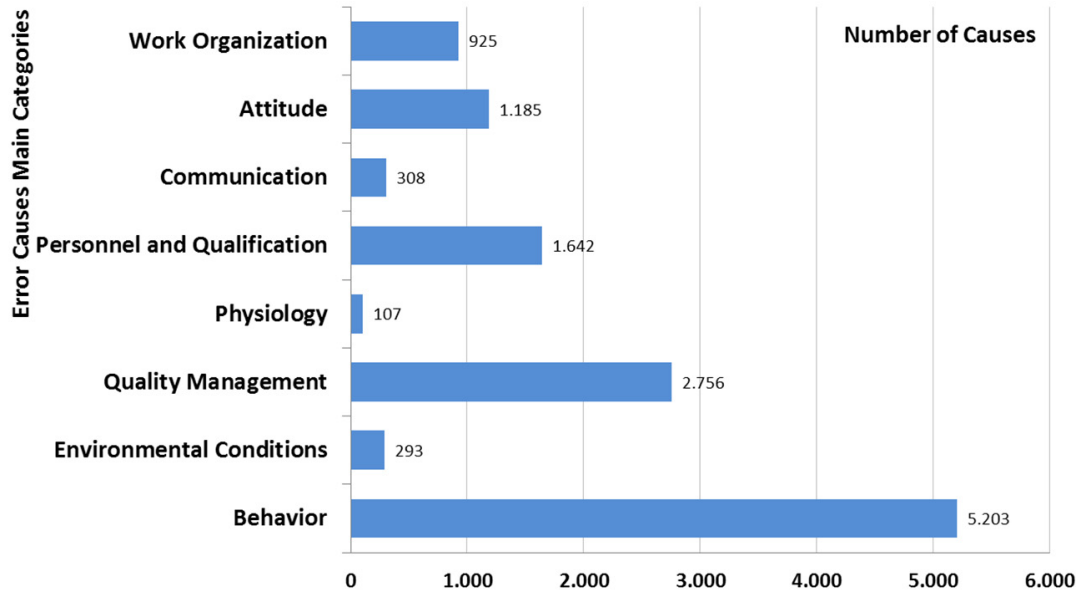

Figure 24: Overview of error causes main categories (Käppler et al. [4]).

strategy, on the other hand, is to gain information on risks and costs so as to allow successful risk management which can be verified by quality control.

Figure 25 shows cost risks of the observed errors of violation of usage regulations, incorrect risk evaluation, playful handling, failing to implement quality assurance measures and incorrect operation and actions. These errors account for almost all cost risks; Nota Bene that simple addition results in more than 100 percent since more than one errors occurred per event. Here, the circle of conventional accident analysis and modern safety management is completed. Indeed, in the case of weapon and munitions handling, errant behavior is also the cause of 90 percent of all accidents. Improvements can certainly be made in this respect by stricter controls on adherence to rules and prohibitions. "Prevention" based on law enforcement and the fear of possible consequences is, however, of little long-term success, as unsuccessful efforts in the past have shown.

This is where a proactive, or even a generic, safety culture comes in. It even specifically avoids the enforcement of rules, to motivate workers to cooperate openly in central error documentation and analysis of causes of error, for example. It goes further, applying their efforts to the causes of these errors. Preventive measures are not developed until insights have been gained.

Figure 26 shows a corresponding overview of cost risks for the eight categories of causes of error in thousands of euro. Behavioral failures are not as expensive as might be expected: They were a factor in 42 percent of incidents, but caused only 34 percent of resulting costs. Similar picture with personnel and qualification failures: Only 9 percent of cost risks were caused, although they occurred in 13 percent of cases. In contrast, attitude failures were only involved in 10 percent, but caused more than double, i.e., 21 percent of total costs. Investment and costs for work organization failures were driving cost risks, too, a factor in 7 percent of the cases but causing 11 percent of cost risks. Quality management failures showed a different result: A factor in 22 percent of incidents, they also caused 19 percent of costs. 


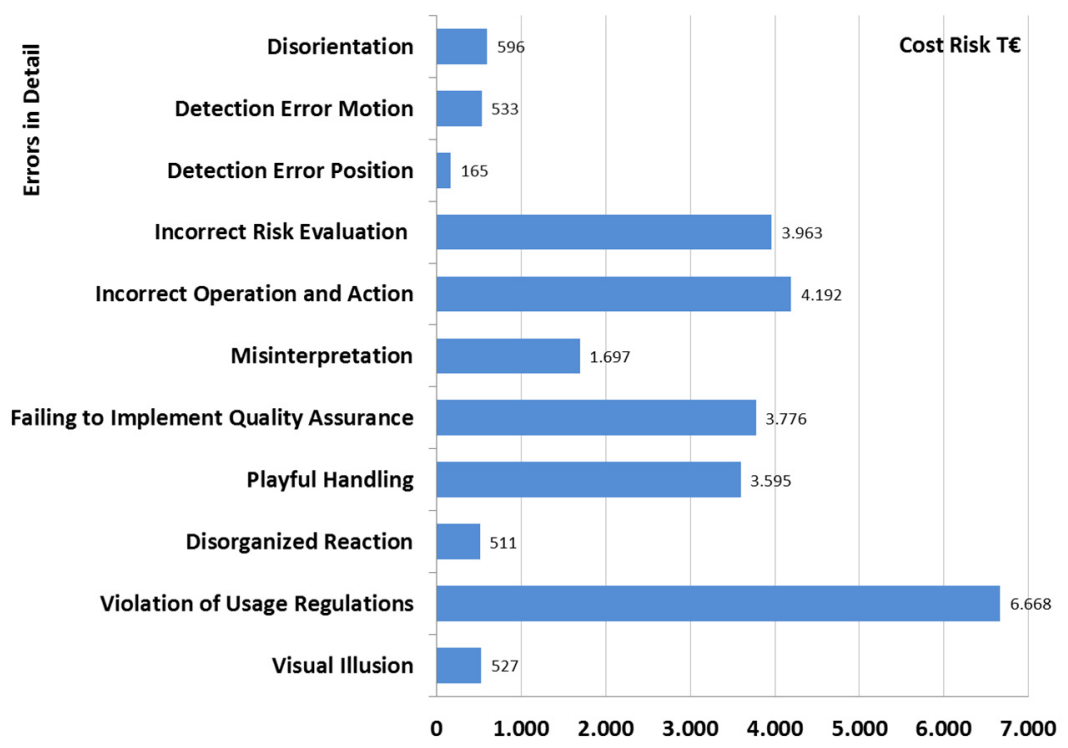

Figure 25: Cost risk of errors in detail in $€ \mathrm{~K}$ (Käppler et al. [4]).

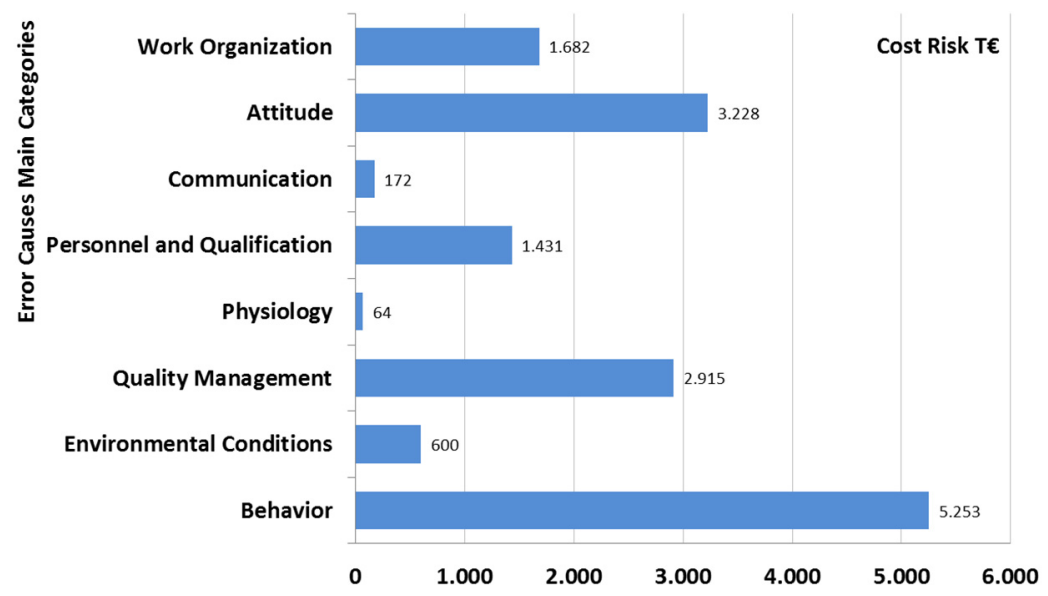

Figure 26: Overview of cost risk of error causes main categories in $€ \mathrm{~K}$ (Käppler et al. [4]).

This comparison shows one significant advantage of risk and cost analyses and allows important decisions to be made: Personnel and qualification failures may have been the second most frequent cause of error, but risk analysis attributes a relatively low cost risk to them. In terms of prevention, this is initially of secondary importance. On the other hand, the results of the statistical analysis for communication failures are confirmed, as are the commonly quoted 
physiology and environmental conditions: Their cost risks are all below 1 percent and for now only come in third place altogether for preventive measures. Based on this strategic decision, detailed information is now required in order to derive concrete preventive measures.

Figure 27 shows the causes of error with the highest direct expenses; costs below $€ 100 \mathrm{~K}$ are not listed, to give a clearer overview.

Forty-four percent of cost risks are incurred by just three causes: Lack of conscientiousness (attitude), deficient supervision and control (quality management) and knowledge deficiencies (behavior). Failures in equipment, practical training, workload and inattentiveness involve markedly lower cost risks; from a preventive point of view they are initially of secondary importance. Preventive measures related to other causes of error come in third place.

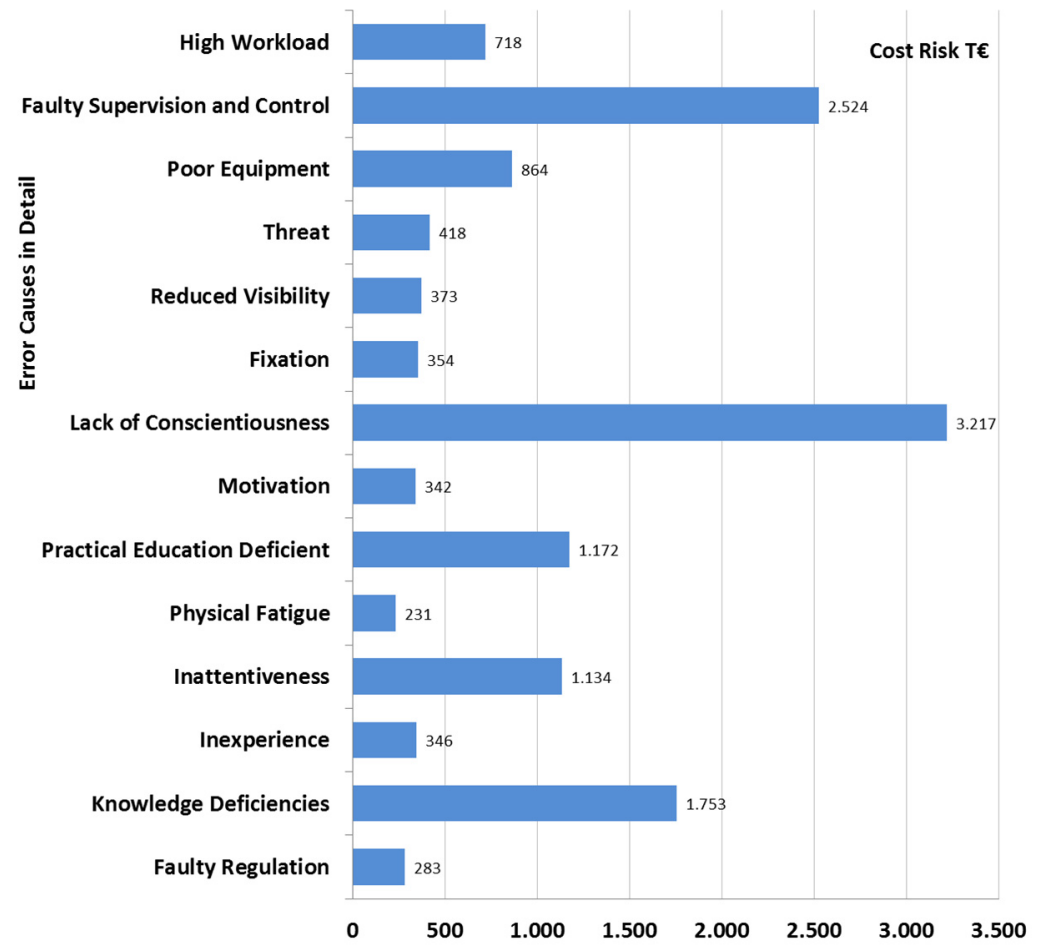

Figure 27: Direct cost risk of error causes in detail in $€ \mathrm{~K}$ (Käppler et al. [4]).

Based on this information, risk management suggests preventive measures, starting with conscientiousness, supervision and control, as well as knowledge gaps. Thus, all that remains is to investigate in detail which knowledge gaps there are, which separate failures in training, procedure or the manuals have caused errors, and which measures can be used to reduce the number of accidents occurring or lessen their results. That task does not fall to this report, but to the hands of experts. Here, there can only be speculation on whether, for example, 
selection processes can already diagnose lack of conscientiousness up front. The same is true of optimization measures for supervision and control.

Interestingly, above all, the trends observed put safety culture at the management level of the German armed forces to the test, as they concern quality assurance, supervision and testing, and responsibility. Altogether, the analysis shows potential savings of more than $€ 10$ million a year just for these three areas.

\subsection{Quality assurance}

Of course, there are also technological failures, as the following example shows: Favored by knowledge gaps (workers' level) and lacking supervision (management level), when dealing with weapons and munitions, acoustic traumas are a frequent injury which can lead to hardness of hearing or even deafness. The hearing protection used today is linear and cuts out all external sounds equally across the whole range of frequencies, including communication, radios or sounds people make themselves. For some exercises, it needs to be removed, even when there is a danger of shots breaking: quite clearly poor equipment.

An analysis of the 2002 data showed that only one in ten acoustic traumas are reported and that in 2002, including this hidden figure, there must have been 2,000 traumas, of which about half were put down to linear hearing protection. In 2002 alone, the costs for sickness and absence were around $€ 2.8$ million.

Non-linear earplugs could be a remedy. The plastic devices defend against thuds and bangs, yet, according to Randow [17] allow the soldiers to perceive their environment at normal sound levels without restriction. Implementing this preventive measure for all soldiers requires a one-off cost of $€ 2.5$ million in total. This can be expected to pay for itself within a year, even without taking into account damage payments, rehabilitation costs or pensions. Consequently, implementation was initiated. Non-linear earplugs are currently in a trial phase.

One last comment on this as regards quality assurance and Swiss cheese, and once again, the circle is completed: As part of the trial, earplugs were originally purchased in the sizes small and large; after removal from the outer packaging, they cannot be told apart from the earplug itself. The soldiers were also rarely aware that different sizes existed at all. Unsurprisingly, acoustic traumas occurred increasingly due to unsuitable or lost earplugs. In the meantime this was remedied by using one flexible standard size for all.

\section{Summary and outlook}

ARIADNE is an integrated safety management system which promotes a proactive safety culture. On a cost-risk basis, it formulates effective preventive measures to improve safety, performance and reliability. It aims to reduce costs while simultaneously improving the protection of lives, health and resources. ARIADNE offers the following advantages: 
- The database's central archive makes all the information, data, documents, reports and analyses available online all over the world, at any time. When there is no internet connection, all documents and data are imported and exported at a later time.

- The safety management system sets standards for data acquisition, investigation, analysis, risk management, prevention and quality assurance of accidents and hazards, with an extensive workflow and intuitive user guidance

- Intricate cost models calculate actual costs resulting from accidents and attribute them in detail to errors and causes

- Risk management creates effective hazard and trend analyses and predicts the risks and costs of all errors and causes

- Risk simulation leads to well-founded decisions about effective preventive measures

- Quality management ensures that the effectiveness of the preventive measures is documented and checked.

ARIADNE is currently deployed by the German armed forces in the field of weapons and munitions and adapted to German Air Force incidents. The results show, for example, that 10 percent of accidents cause 90 percent of the resulting costs. Causes of error from the "behavior" category accrue the highest costs.

ARIADNE is based on Internet technology, is used as an Internet or intranet application and operated using an Internet browser. It does not require the installation of software or clients. This total lack of proprietary platforms and formats offers considerable financial advantages. ARIADNE is available in German and English as a complete safety management system or service, and can be applied to a wide range of working activities. More information is provided on www.ariadne-sms.com.

\section{References}

[1] Bibliographisches Institut \& F. A. Brockhaus AG, 2007.

[2] Reason J. Human Error. Cambridge, Mass: University Press, 1990.

[3] Senders J. \& Moray N. (1991) Human Error. Hillsdale, NJ: Erlbaum, 1991.

[4] Käppler W.D., Pressler R., Siebel M. \& Specht D. Smart Safety Management mit ARIADNE SMS. Berlin Heidelberg: Springer Vieweg, 2014.

[5] Käppler W.D. Menschliche Fehler als Unfallursachen: Untersuchungen und Ergebnisse mit ARIADNE. In Grandt, M (Ed) Verlässlichkeit der Mensch-Maschine-Interaktion. Bonn: Deutsche Gesellschaft für Luft- und Raumfahrt eV, Report 04-03, pp. 197-212, 2004.

[6] Käppler W.D. Smart Driver Training Simulation. Save Money. Prevent. Berlin Heidelberg: Springer, 2008.

[7] Frieling E. \& Sonntag K. Lehrbuch Arbeitspsychologie. Bern: Huber, 1999. 
[8] Chappelow J.W. Causal Factors in Military Aircraft Accidents: Study Outline. Farnborough: DERA/CHS/MID/TR980168, 1998.

[9] Rohrmann B. Empirische Studien zur Entwicklung von Antwortskalen für die sozialwissenschaftliche Forschung. Z.f. Sozialpsychologie 9, pp. 222245, 1978.

[10] Käppler W.D. Beitrag zur Vorhersage von Einschätzungen des Fahrverhaltens. Düsseldorf: VDI Fortschrittsberichte 12/198, 1993.

[11] Käppler W.D., Pressler R. \& Specht D. Sicherheitsmanagement mit ARIADNE. In Ergonomie und Mensch-Maschine-Systeme (pp. 437-462, Eds Schmidt, Schlick, Grosche) Heidelberg, Berlin, New York: Springer Vieweg, 2008.

[12] INSAG Safety Culture. Vienna: IAEA Safety Series 75-INSAG-4, 1991.

[13] Hudson P. Applying the Lessons of High Risk Industries to Health Care. Qual. Saf. Health Care 12, pp. 7-12, 2003.

[14] Morgenstern C. Die unmögliche Tatsache. In Gesammelte Werke. Munich: Piper, 1910, edn. 2004.

[15] Käppler W.D. \& Dalinger E Derivation of Safety Measures through Human Error Risk Analyses. In C.A. Brebbia et al. (Eds.) Safety and Security Engineering. Southampton, Boston: Witpress, pp. 253-263, 2005.

[16] Käppler W.D. Sicherheit im Motorradhandel. Bonn: BG Handel und Warendistribution FB 1, 2008.

[17] v. Randow G. Stöpsel und Kanonen. Frankfurter Allgemeine Sonntagszeitung 10, p. 59, 2003. 